\title{
Estudio faunístico de los mosquitos (Díptera, Culicidae) de la comarca del Somontano de Barbastro y su posible relevancia en la difusión del paludismo
}

\author{
Rubén Bueno-Marí \\ Laboratorio de Entomología y Control de Plagas, Instituto Cavanilles de Biodiversidad y Biología Evolutiva, Universitat de \\ València. C/ Catedrático José Beltrán, 2. 46980. Paterna (Valencia). \\ Departamento de Ciencias Naturales, Instituto de Estudios Riojanos (IER).
}

\begin{abstract}
Correspondencia
R. Bueno-Marí

E-mail: ruben.bueno@uv.es

TIf.:+34868 884961
\end{abstract}

Recibido: 5 agosto 2013

Aceptado: 29 noviembre 2013

Publicado on-line: 20 diciembre 2013

\section{Resumen}

Se han capturado 1.106 ejemplares larvarios de culícidos pertenecientes a 12 especies en la Comarca del Somontano de Barbastro, de las cuales dos (Culex impudicus y $C x$. territans) se citan por primera en la provincia de Huesca. Pese a que la especie más abundante fue $C x$. pipiens, desde un punto de vista epidemiológico señalar la elevada presencia de mosquitos del género Anopheles, potenciales vectores de la malaria, en diversas regiones de la comarca. Especialmente relevante es la destacada densidad que presenta An. atroparvus, importante vector palúdico, en biotopos concretos de la región más meridional del área de estudio. Adicionalmente, también se han detectado especies muy antropofílicas, como Cx. modestus u Ochlerotatus caspius, que pueden ocasionar episodios molestos para la población humana circundante a sus criaderos larvarios. En definitiva, se aporta información bioecológica, biogeográfica y epidemiológica de las capturas de este grupo de insectos de máximo interés sanitario en la Comarca del Somontano.

Palabras clave: Vectores de enfermedades, Paludismo, Control de plagas, Entomología médica, Salud pública, Biodiversidad.

\begin{abstract}
Faunistic study of mosquitoes (Diptera, Culicidae) in Somontano de Barbastro and its possible relevance on malaria spread

This research has allowed the collection of 1106 larval exemplars of culicid mosquitoes and the identification of 12 species in the Region of Somontano de Barbastro (Northeastern Spain). Two of twelve species collected, Culex impudicus y Cx. territans, are firstly reported from the Province of Huesca. Although $C x$. pipiens has been the most abundant species of the study, from an epidemiological point of view it must be noted that some species of Anopheles genus were detected in high densities. Especially interesting were some the findings of the main malaria vector in Europe, An. atroparvus, which even was the dominant species in several environments. Moreover, information about biotopes of higly antropophylic species responsible of potential nuisances to human population, like Cx. modestus or Ochlerotatus caspius, are also provided.
\end{abstract}

Key words: Disease vectors, Malaria, Pest control, Medical entomology, Public health, Biodiversity. 


\section{Introducción}

Los mosquitos culícidos (Diptera, Culicidae) son los vectores de transmisión biológica de algunas de las enfermedades más importantes a nivel de la Salud Pública mundial. Parasitosis como el paludismo/malaria o la filariosis, y arbovirosis como el dengue, la fiebre amarilla, los Virus del Nilo Occidental o de la fiebre de Chikungunya son ejemplos suficientemente explícitos del fuerte impacto de los culícidos sobre la salud humana.

La enfermedad parasitaria más importante del mundo, la malaria o paludismo, supone uno de los mejores exponentes para estudiar la vulnerabilidad de las enfermedades emergentes o reemergentes de transmisión vectorial en nuestro país. En la actualidad se diagnostican en España alrededor de 400 casos de paludismo anualmente. En todos los casos se trata de paludismo importado, es decir, se debe a personas inmigrantes y turistas procedentes de zonas endémicas que llegan parasitados a nuestro país. Al respecto, la concienciación o la legislación que obligue a tomar las medidas profilácticas necesarias a los turistas o inmigrantes residentes en España que decidan viajar a zonas de riesgo por enfermedades infecciosas transmisibles, son sin duda algunas de las cuestiones a mejorar y potenciar. Sin embargo, todas estas coyunturas serían meras anécdotas epidemiológicas si no fuese porque en España seguimos teniendo, tal y como sucedía en el pasado, excelentes vectores de la enfermedad. Es decir, la receptibilidad palúdica es relativamente elevada, sobre todo en ciertas regiones rurales donde existen destacadas densidades del principal vector europeo de la parasitosis, Anopheles atroparvus Van Thiel, 1927 (Bueno-Marí \& Jiménez-Peydró 2010). En general, los hábitos bioecológicos de los vectores apuntan a que, al menos en nuestro país y en la práctica totalidad del continente europeo, el paludismo es una enfermedad eminentemente rural. No obstante, esta tesitura podría revertirse por completo si, fruto de la globalización, se produjese la llegada de vectores africanos cuya distribución puede ser común en ambientes urbanos como Anopheles gambiae Giles, 1902 o Anopheles arabiensis Patton, 1905, gracias a su adaptabilidad para completar el desarrollo larvario en pequeños recipientes domésticos (Bueno-Marí et al. 2009a).

Aun teniendo en cuenta todas las cuestiones previamente descritas, cabe mencionar que recien- temente se ha vuelto a detectar en España, concretamente en una zona rural de la provincia de Huesca (en la Comarca de los Monegros), el primer caso de paludismo autóctono desde hace 50 años. La paciente fue una mujer de mediana edad (44 años) que, a priori, no representaba ninguno de los grupos de riesgo (no viajó a zonas de endemia, no recibió transfusiones sanguíneas de ningún tipo, ni estuvo en los últimos meses en las proximidades de ningún aeropuerto internacional). Asimismo, cabe destacar la confirmación del infradiagnóstico del paludismo importado en nuestro país, ya que en la provincia de Huesca no se había reportado ningún caso en los últimos años. El protozoo implicado fue Plasmodium vivax Grasi and Feletti 1890 (Santa-Olalla Peralta et al. 2010) y todos los indicios apuntan a que fue $A n$. atroparvus (Bueno-Marí et al. 2012a) el vector de transmisión.

El objetivo del trabajo aquí presentado es doble. En primer lugar, actualizar la diversidad faunística de los mosquitos culícidos de la provincia de Huesca, tomando como área de estudio la Comarca del Somontano de Barbastro y teniendo en cuenta que existen citadas un total de 64 especies en España, de las cuales algunas se consideran actualmente erradicadas de nuestro país (Bueno-Marí et al. 2012b). Y en segundo lugar, analizar la receptibilidad palúdica de dicha comarca en función de los datos derivados de los anofelinos capturados a partir de intensivos muestreos larvarios en el área de estudio. Como se ha mencionado con anterioridad, este último objetivo es de mayor interés si cabe, tras el acontecimiento en la vecina Comarca de Los Monegros del primer caso de paludismo autóctono en España en los últimos 50 años.

\section{Breve sinopsis histórica del paludismo en el Somontano de Barbastro}

Los episodios epidémicos de paludismo fueron relativamente frecuentes en la Comarca del Somontano de Barbastro a finales del siglo XVIII. Entre 1783 y 1785 , el paludismo causó centenares de muertos con dos regiones claramente heterogéneas con respecto a la morbi-mortalidad de la enfermedad (Nieto Callén \& Bosch Ferrer 1991): por una parte las zonas de baja topografía bañadas por el río Cinca y dedicadas a diversos tipos de cultivo, con elevada incidencia de la parasitosis, y por otra parte, las zonas montañosas de la sierra y 
terrenos secos somontanos del sur de la comarca, donde la enfermedad apenas tiene incidencia. El río Cinca era un foco de cría óptimo y habitual para An. atroparvus. Si a esta situación le añadimos el hecho de que, coincidiendo con los episodios epidémicos del siglo XVIII se produjo un importante descenso de la actividad ganadera en zonas circundantes al río debido a destacados periodos de sequía, resulta evidente que los anofelinos allí presentes, principalmente An. atroparvus, comenzaron a alimentarse de forma más activa sobre el ser humano dada la ausencia de ganado que podía, en parte, desviar las preferencias hematofágicas de las hembras de Anopheles. Por el contrario, en regiones montañosas del norte de la comarca, la abundancia de otros posibles hospedadores para las hembras de mosquitos y la baja densidad humana, posibilitaron que la enfermedad no alcanzase los mismos grados de severidad.

\section{Material y Métodos}

Para el muestreo de larvas de mosquitos, se han seleccionado aquellos biotopos hídricos más relevantes de la comarca. Dada la gran diversidad de colecciones hídricas de la comarca, se han elegido aquéllas que presentan características más adecuadas para la proliferación de mosquitos. En general, los culícidos exigen de aguas lénticas o de escasa corriente para el desarrollo larvario. En consecuencia, se han muestreado los márgenes remansados de los cursos fluviales de la comarca (Alcanadre, Isuala, Vero y Cinca) en distintos tramos, así como las numerosas fuentes, balsas, abrevaderos y pozas presentes en diversos puntos, entre los que destacan los incluidos en el Parque Natural de la Sierra y Cañones de Guara. Dadas las connotaciones directas de este estudio para la población humana, también se han visitado microambientes hídricos que pueden albergar larvas de mosquitos en ambientes urbanos, como fuentes ornamentales, imbornales sinfónicos, recipientes domésticos, etc.

En total, se han estudiado 47 localidades (Fig. 1) cuyos datos de localización se pueden consultar en el Apéndice, donde aparece el total de capturas por especie.

Los muestreos se llevaron a cabo entre los meses de junio a octubre de 2011, periodo que coincide con el rango temporal ideal para el desarrollo poblacional de los mosquitos. La técnica emplea- da para la recolección de estados inmaduros es la conocida como "dipping", consistente en la introducción de un recipiente de $350 \mathrm{ml}$ de capacidad en el medio hídrico del cual se desee obtener una alícuota. En aquellos focos de cría de reducidas dimensiones donde no es posible introducir el dipper (cubos, bidones, charcos someros, etc.), los muestreos se realizaron directamente mediante el empleo de una pipeta tipo Pasteur, seleccionando de manera individual los estadíos inmaduros recolectados. El esfuerzo muestral se fijó en 10 minutos que incluyen la búsqueda y captura de larvas en cada punto de muestreo; tiempo que se ha demostrado como suficiente para la obtención de datos cualitativos de relevancia en muestreos que pretenden abarcar amplias áreas de estudio (Bueno-Marí 2010).

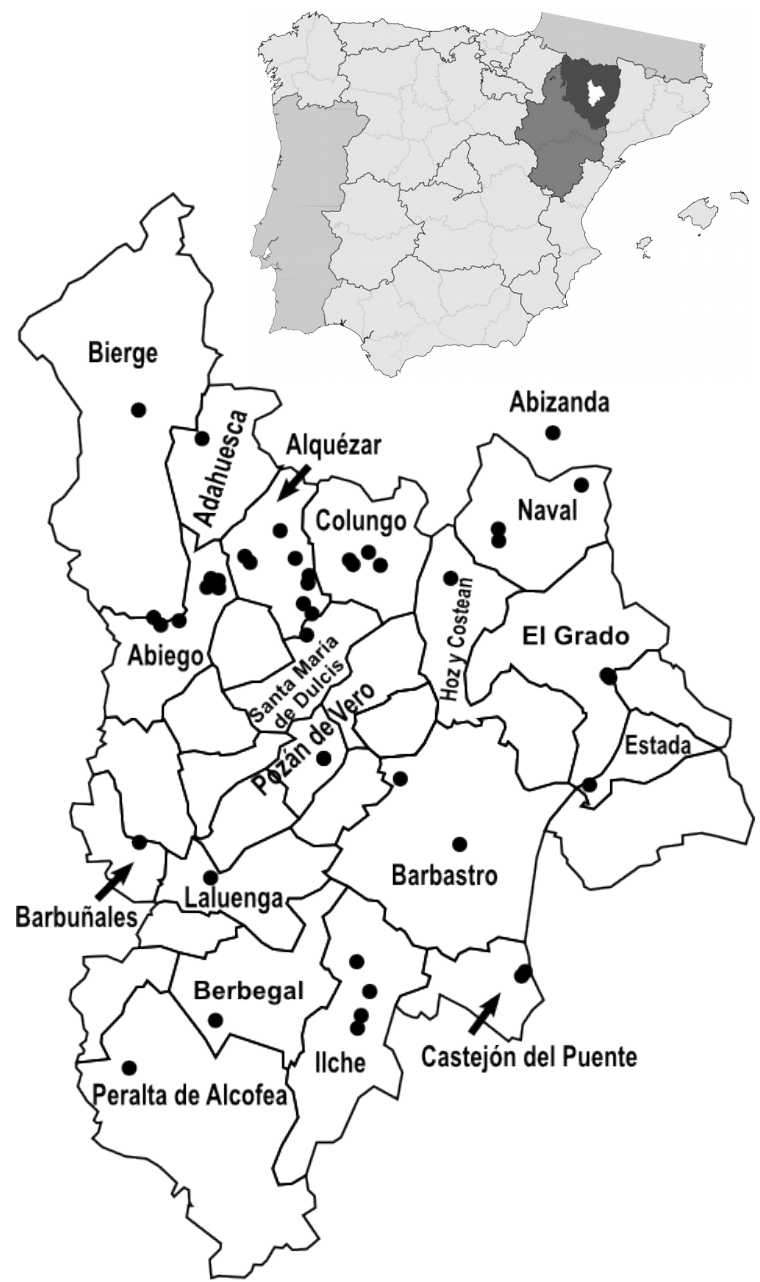

Figura 1. Mapa de las localidades estudiadas, situadas respecto a los municipios de la comarca del Somontano de Barbastro, provincia de Huesca.

Figure 1. Map of studied localities, framed in the municipalities of Somontano de Barbastro natural region, Province of Huesca. 
La determinación específica de los ejemplares larvarios capturados tuvo lugar tras el análisis bajo lupa binocular de la anatomía, morfología y morfométrica larval siguiendo las claves taxonómicas pertinentes (Romi et al. 1997, Darsie \& Saminadou Voyadjoglou 1997, Schaffner et al. 2001, Bueno-Marí 2010).

\section{Resultados y discusión}

\section{Análisis faunístico, bioecológico y biogeo- gráfico de las especies capturadas}

Los intensivos muestreos han permitido la captura e identificación de un total de 1.106 ejemplares larvarios de mosquitos pertenecientes a las siguientes 12 especies (Tabla 1), de las cuales $\mathrm{Cu}$ lex impudicus Ficalbi, 1890 y Culex territans Walker, 1856 son citadas por primera vez en la Provincia de Huesca (Encinas Grandes 1982). Información más detallada acerca de las capturas puede consultarse en el Apéndice.

\begin{tabular}{lc}
\hline \multicolumn{1}{c}{ Especie } & $\mathbf{N}^{\circ}$ ejemplares \\
\hline Anopheles atroparvus & 166 \\
Anopheles maculipennis & 42 \\
Anopheles petragnani & 27 \\
Culex hortensis & 23 \\
Culex impudicus & 27 \\
Culex mimeticus & 67 \\
Culex modestus & 9 \\
Culex pipiens & 477 \\
Culex territans & 85 \\
Culiseta annulata & 4 \\
Culiseta longiareolata & 131 \\
Ochlerotatus caspius & 48 \\
\hline
\end{tabular}

Tabla 1. Especies capturadas y número de ejemplares.

Table 1. Collected specimens for each species.

\section{Anopheles atroparvus Van Thiel, 1927}

Especie de distribución paleártica ampliamente diseminada por toda Europa (Schaffner et al. 2001). Se considera el vector palúdico más establecido en el viejo continente y, por tanto, el mejor adaptado a las condiciones climatológicas y geoantrópicas aquí existentes (Bueno Marí \& Jiménez Peydró 2008). En España, la coincidencia de las primeras generaciones de hembras post-hibernantes, junto con la emergencia de las recidivas tardías de $P$. vivax, era una de las principales causas del sostenimiento de la endemia palúdica en diversos territorios (Lozano 1953). Salvo en la Región del
Bajo Segura (sureste de la provincia de Alicante-este de la provincia de Murcia), donde otra especie, denominada Anopheles labranchiae Falleroni, 1926, monopolizaba claramente los episodios de transmisión de malaria en la primera mitad del siglo XX (Gil Collado, 1940), lo cierto es que en el resto del territorio peninsular era $A n$. atroparvus el principal vector responsable de la parasitosis.

Los biotopos larvarios habituales de An. atroparvus son pequeñas lagunas, encharcamientos temporales, márgenes remansados de ríos, canales $\mathrm{y}$ arrozales, todos ellos caracterizados por aguas poco eutrofizadas y dulces u oligosalobres (López Sánchez 1989); pese a que de manera esporádica también se han descrito capturas en aguas salobres de hasta $8 \mathrm{gr} / \mathrm{l}$ (Schaffner et al. 2001). Las capturas realizadas en la Comarca del Somontano de Barbastro se corresponden a la perfección con los datos teóricos previamente expuestos, ya que la mayoría de hallazgos se caracterizaron por una baja salinidad del foco hídrico, oscilando ésta entre 0,2 y $0,7 \mathrm{gr} / 1$, pese a que se llegó a detectar larvas de la especie a una salinidad de 5,4 y 6,4 gr/l, en un ambiente fluvial de la zona de El Salinar (Naval) y charcos temporales en zonas de maizales de Ilche, respectivamente. Particularmente interesantes, por la elevada densidad poblacional encontrada, son los hallazgos en diversos puntos del río Cinca, así como en una pequeña extensión dedicada al cultivo del arroz situada en la pedanía de Monesma (Ilche).

Por último, indicar que la capacidad vectorial de An. atroparvus no se limita exclusivamente a la malaria, sino que también puede transmitir virus como el Virus del Nilo Occidental, Batai, Tahyna, así como participar activamente en enfermedades como la filariasis canina y la tularemia (Schaffner et al. 2001).

\section{Anopheles maculipennis Meigen, 1818}

Especie distribuida a nivel paleártico y presente en la mayoría de países europeos Schaffner et al. 2001). Se trata de una especie muy próxima morfológica y taxonómicamente a An. artroparvus. Cuando comenzó a seccionarse el "complejo multiespecifico maculipennis", se postulaba que, a diferencia con An. atroparvus, la especie An. maculipennis picaba de manera más infrecuente al hombre, ya que solía habitar en regiones más elevadas y menos antropizadas, debido a su marcada 
preferencia por aguas más frías y menor tolerancia a la eutrofia y la salinidad del agua (Hackett \& Missiroli 1935).

Nuestros hallazgos en la Comarca del Somontano de Barbastro se sitúan en torno a esta tesitura, ya que siempre se encontró a An. maculipennis en aguas de escasa salinidad y preferentemente alejadas de ambientes antropizados. Todos los focos de cría se han localizado en ambientes fluviales de escasa salinidad, oscilando ésta entre los 0,2 y $0,4 \mathrm{gr} / 1$.

An. maculipennis es una especie multivoltina, eurígama, que hiberna en estado imaginal y cuyas hembras pueden transmitir, además de plasmodios de afección humana, dirofilarias y virus como el Virus del Nilo Occidental, Batai y Tahyna.

Anopheles petragnani Del Vecchio, 1939

Especie de distribución paleártica que se circunscribe al mediterráneo occidental (Schaffner et al. 2001). Exhibe dos o tres generaciones anuales, con picos poblacionales en abril, julio y septiembre, y existe bastante controversia acerca de si la hibernación es larvaria o imaginal (Encinas Grandes 1982).

Se trata del anofelino menos abundante en el área de estudio y que fue hallado exclusivamente en encharcamientos temporales, antiguos lavaderos, fuentes ornamentales y abrevaderos.

Es una especie autógena, estenógama, exofágica y zoofílica, picando preferentemente a animales domésticos (Lachmajer 1971) y únicamente al hombre de forma accidental y en las inmediaciones de sus biotopos larvarios (Encinas Grandes 1982). Hasta el momento, se desconoce su posible implicación en la diseminación de agentes patógenos.

\section{Ochlerotatus caspius (Pallas, 1771)}

Especie paleártica muy abundante en zonas pantanosas halófilas (Edwards 1921, Rioux 1958), gracias a su destacada resistencia frente a la salinidad del agua, pudiendo incluso desarrollarse en ambientes hipersalinos por efecto de la evaporación, con concentraciones extremas de hasta $106 \mathrm{gr} / 1$ (López Sánchez 1989). También pueden criar en aguas dulces albergadas en charcas temporales y prados inundados (Encinas Grandes 1982).

En la comarca del Somontano de Barbastro únicamente se consiguió capturar en una ocasión en Permisán (Ilche); concretamente en encharca- mientos salobres situados en campos de cultivo de maíz y debidos al riego de dicho cultivo. Es más que probable que Oc. caspius sea una especie relativamente común en el sur de la comarca, debido a la abundancia en la zona de estos pequeños encharcamientos que permiten la eclosión de los huevos que la especie, como el resto de aedinos, deposita directamente sobre la superficie del sustrato seco o húmedo potencialmente inundable.

Su capacidad vectorial es extensa, englobando a filarias (Aranda et al. 1998) y arbovirus como el Virus del Nilo Occidental o Tahyna (Schaffner et al. 2001).

\section{Culex modestus Ficalbi, 1889}

Especie presente a nivel paleártico (fundamentalmente en la Europa meridional) y Oriental (Moussiegt 1990). Las larvas suelen aparecer en arrozales, canales y zonas de inundación temporal (Gil Collado 1930) entre los meses de mayo y octubre (Moussiegt 1990). Las hembras de Cx. modestus son exofílicas, exofágicas, hibernantes y raramente se alejan más allá de los 200 metros desde sus criaderos larvarios (Minar 1969, Mouchet et al. 1970). Si el hombre se encuentra en posiciones cercanas a los focos de cría, es picado ávidamente durante las horas diurnas y, en menor medida, también al anochecer (Guille 1976).

De manera similar al caso de Oc. caspius, la especie $C x$. modestus sólo ha sido hallada en encharcamientos temporales de carácter halófilo situados en el sur de la comarca, en este caso en Monesma (Ilche) .

Cx. modestus se ha asociado a la posible transmisión de la filariasis canina, la mixomatosis y los virus Tahyna, Batai, Sindbis, Lednice y Virus del Nilo Occidental (Chippaux et al. 1950, Hannoun 1971, Schaffner et al. 2001).

\section{Culex mimeticus Noè, 1899}

Especie distribuida por la región Paleártica, circunscrita a la subregión Mediterránea, y el sur de la región Oriental (Schaffner et al. 2001). En la región Oriental, la especie exhibe una marcada preferencia orofílica, con hallazgos hasta los 3055 metros de altitud en el Tibet (Feng 1938). En clara sintonía con otros autores (Gil Collado 1930, Encinas Grandes 1982), hemos observado una clara propensión de $C x$. mimeticus por ovipositar en márgenes remansados de ríos y arroyos, así como en receptáculos de origen antrópico de grandes di- 
mensiones como pozos, balsas y abrevaderos.

Las hembras son el estado hibernante habitual de esta especie multivoltina cuya biología, pese a ser poco conocida, parece girar en torno a la presencia colindante de aves, debido a que éstas suelen ser su hospedador preferente (Schaffner et al. 2001), sin obviar ciertos episodios endofágicos, totalmente excepcionales, en viviendas humanas (Sicart, 1951). Se han encontrado poblaciones de Cx. mimeticus portadoras del Virus del Nilo Occidental.

\section{Culex pipiens Linnaeus, 1758}

Especie distribuida por toda la región Holártica, el este y sudeste de África, y Sudamérica (Stone et al. 1959). También es conocido como el "mosquito común", dada su absoluta ubicuidad. Es multivoltina, pudiendo completar hasta 6 ciclos gonotróficos y, pese a que las hembras son las que hibernan habitualmente, también se han descrito casos de quiescencia larvaria, sobre todo, en regiones más cálidas (López Sánchez 1989).

Durante los muestreos se capturaron larvas de $C x$. pipiens en multitud de biotopos de diferente fisiografía y dispar físico-química, como abrevaderos, balsas, ambientes fluviales, encharcamientos rocosos, canales de aguas fecales, bidones de riego, etc., siendo en consecuencia la especie más abundante y distribuida en el presente estudio.

Su adaptabilidad a los ambientes urbanos, unida a su elevada endofilia, le permite ser la especie más frecuente en el interior de las viviendas (Llave Correas \& González Mora 1996), picando ávidamente al ser humano durante la noche. Es capaz de vehicular numerosos arbovirus, tales como el Virus del Nilo Occidental, Sindbis, Tahyna, Batai y Usutu, además de filarias como Dirofilaria immitis (Leidy, 1856) y plasmodios de afección aviar (Aranda et al. 1998, Schaffner et al. 2001, Busquets et al. 2008).

Debido a que la identificación de todo el material entomológico capturado se ha producido a partir del estudio de formas larvarias, la posible presencia de especies vicariantes de zonas montañosas como Culex torrentium Martini, 1925 no pudo certificarse, al no analizarse en detalle un claro elemento diagnóstico diferencial de la especie como es la morfología de la genitalia de los machos. No obstante, esta cuestión queda pendiente de revisarse en futuros muestreos en la zona, dado que diversos datos parecen indicar que
$C x$. torrentium es una especie relativamente abundante en la zona pirenaica (Aranda et al. 2000).

Culex hortensis Ficalbi, 1889

Especie paleártica de distribución amplia por toda Europa salvo las regiones más norteñas del continente (Schaffner et al. 2001). Sus biotopos larvarios son de muy diversa tipología y suelen desarrollarse en aguas poco eutrofizadas y dulces, aunque también se han observado en ambientes salobres (López Sánchez 1989). Las poblaciones peninsulares de Cx. hortensis parecen mostrar una tendencia orofílica (Gil Collado 1930, Rioux 1958, Bueno Marí et al. 2009b). Pese a que $C x$. hortensis es una especie relativamente frecuente en la zona pirenaica (Bueno Marí et al. 2009b), en la Comarca del Somontano de Barbastro únicamente se ha conseguido capturar de manera aislada.

Es multivoltina, hiberna en estado imaginal, se alimenta preferentemente sobre reptiles y batracios (Schaffner et al. 2001) y aún se desconoce mucho acerca de su ciclo fenológico.

Culex impudicus Ficalbi, 1890

Especie propia de la Región Paleártica que se distribuye únicamente en los países mediterráneos y cuya escasa información disponible, indica que presenta una clara tendencia por aguas limpias, frías, umbrías y dulces (Schaffner et al. 2001). Las hembras se encargan de la supervivencia de la especie durante el invierno $\mathrm{y}$, al transcurrir éste, parece que se alimentan preferentemente de batracios, ignorando casi por completo al ser humano (Rioux 1958). Nosotros la hemos hallado fuertemente asociada a los remansos de ríos, arroyos y encharcamientos permanentes, y siempre cohabitando con Cx. territans Walker, 1856, situación, ésta última, que también ha sido advertida por otros autores (Encinas Grandes 1982, Bueno Marí 2010) y que iría en contradicción con la teoría propuesta de que $C x$. impudicus fuese, en realidad, un grupo vicariante meridional de Cx. territans (Rioux 1958).

Es multivoltina, eurígama, se desconoce su posible papel vectorial $\mathrm{y}$, según los datos disponibles, jamás había sido citada hasta ahora en la provincia de Huesca.

\section{Culex territans Walker, 1856}

Especie propia de la región Holártica que se en- 
cuentra ampliamente representada en el continente europeo (Horsfall 1972). Los criaderos larvarios también son prácticamente idénticos a los de $C x$. impudicus, si bien $C x$. territans parece adaptarse mejor a los biotopos de grandes dimensiones (Jordá Llona et al. 1993). Se trata de una especie típicamente asociada a ambientes montañosos (Schaffner et al. 2001).

Es una especie multivoltina, eurígama y cuyas hembras hibernan en cavidades tanto naturales como antrópicas, pican a reptiles y anfibios, $\mathrm{y}$ transmiten filarias de afección en batracios (Encinas Grandes 1982, Schäefer 2004). Al igual que sucede con $C x$. impudicus, la ausencia de datos acerca de su captura en la provincia de Huesca, permite indicar que se trata de una nueva cita para la provincia.

\section{Culiseta longiareolata (Macquart, 1838)}

Especie de distribución paleártica, oriental y afrotropical (Schaffner et al. 2001). En Europa su presencia es más común en los países mediterráneos, siendo excepcionales sus hallazgos en regiones más norteñas del continente. Es multivoltina, estenógama, autógena, sus hábitats larvarios son muy variados y, mientras en regiones templadas hiberna en estado larvario, en áreas más frías son las hembras quienes se encargan de la supervivencia de la especie durante el periodo desfavorable (Encinas Grandes 1982, Schaffner et al. 2001).

En la Comarca del Somontano de Barbastro hemos capturado larvas de Cs. longiareolata, en todos los casos, en grandes recipientes hídricos, como bidones de riego o bebederos artificiales. En sintonía con Encinas Grandes (1982) y López Sánchez (1989), debemos indicar la tolerancia de aguas contaminadas que presenta la especie, sobre todo en situaciones domésticas y peridomésticas donde, por otra parte, Cs. longiareolata se presenta asiduamente tal y como se ha constatado en el presente estudio. Teniendo en cuenta todas estas cuestiones, existe bastante consenso en afirmar el hecho de que Cs. longiareolata pueda completar sus ciclos bióticos incluso durante todo el año, siendo esta situación únicamente alterable por la aparición de bajas temperaturas que impondrían la quiescencia larvaria (Romi et al. 1997).

Sus preferencias hematofágicas parecen dirigirse hacia las aves, aunque ocasionalmente piquen también al hombre, y pueden transmitir el paludismo aviar y el Virus del Nilo Occidental
(Schaffner et al. 2001).

\section{Culiseta annulata (Schrank, 1776)}

Especie de distribución paleártica y afrotropical (Edwards 1921, Stone et al. 1959). Es multivoltina, presenta una gran variabilidad de hábitats larvarios, siendo éstos tanto naturales como artificiales, y tolera aguas con amplios rangos de eutrofización y salinidad (López Sánchez 1989, Schaffner et al. 2001). La única captura de la especie en la comarca, tuvo lugar en una balsa en las proximidades de un antiguo lavadero abandonado en la pedanía de Radiquero (Alquézar).

Cs. annulata es zoofílica, picando preferentemente a aves, pero también de manera ocasional al hombre (Encinas Grandes 1982, Alten et al. 2001). Aunque sus hábitos son exofílicos (Schaffner et al. 2001), existen hallazgos en el interior de viviendas (Encinas Grandes 1982), sobre todo si sus criaderos larvarios son domésticos o peridomésticos. Puede transmitir el virus Tahyna y el de la mixomatosis, así como el paludismo aviar (Schaffner et al. 2001).

\section{Receptibilidad del paludismo}

El presente estudio ha permitido la captura de tres especies potencialmente vectores del paludismo en la Comarca del Somontano de Barbastro. Entre estos potenciales vectores, destaca la especie An. atroparvus, ya que es uno de los principales transmisores de la malaria en el continente europeo, y además se trata del anofelino más abundante y ampliamente distribuido en la comarca. Los principales criaderos de la especie han sido los márgenes remansados y encharcamientos temporales de los ríos Cinca, Vero y Alcanadre. Particularmente relevante, por la abundancia de pequeñas acumulaciones discontinuas de agua en los meses veraniegos, es el río Cinca. Sin duda, se trata del criadero larvario de An. atroparvus de mayor extensión e importancia de la comarca. Sin embargo, tal y como se ha expuesto con anterioridad, únicamente posibilita un desarrollo poblacional notable de la especie en época de escaso caudal fluvial, fundamentalmente durante los meses veraniegos.

Cabe destacar que los biotopos larvarios de An. atroparvus no quedan circunscritos exclusivamente al curso fluvial del río, sino que se extienden en función del aprovechamiento hídrico del río por parte del hombre (canales y acequias de 
irrigación, balsas) y por tanto abarcan un perímetro mayor. Pese a que, de forma puntual, An. atroparvus también se ha detectado en los ríos Vero y Alcanadre, el segundo territorio o área de mayor importancia como criadero de la especie lo encontramos en el sur de la comarca. Si bien es cierto que éste es el territorio más seco de la región estudiada, de nuevo debemos fijarnos en acciones antrópicas relacionadas con el aprovechamiento hídrico para entender la situación. Además de la presencia de antiguos lavaderos, el intensivo cultivo del maíz y de la alfalfa ha provocado el desarrollo de abundantes balsas y canales de riego donde ha conseguido asentarse An. atroparvus. De forma especial cabe resaltar la elevada presencia de An. atroparvus, incluso en densidades epidemiológicamente relevantes, en pequeñas extensiones dedicadas al cultivo del arroz situadas en el sur de la comarca.

La segunda especie capturada de mayor importancia en la posible transmisión palúdica es $A n$. maculipennis. Las preferencias ovipositorias de An. maculipennis están muy próximas a An. atroparvus, si bien la primera se inclina habitualmente por aguas más frías y con menor eutrofización y salinidad, con lo que suele desarrollarse en áreas más alejadas de los núcleos poblacionales humanos. Éste puede ser un óptimo factor explicativo para las eventuales acciones hematofágicas que tienen lugar únicamente sobre el hombre, cuando éste penetra en las inmediaciones de los biotopos larvarios de An. maculipennis. En el presente estudio siempre se ha detectado a An. maculipennis en los cursos fluviales de los ríos Cinca, Vero, Alcanadre e Isuala. Dado que es una especie fundamentalmente zoofílica y que sus biotopos larvarios destacan por encontrase en zonas agrestes, su posible interés palúdico en la comarca es prácticamente nulo. En términos similares podemos referirnos a An. petragnani. Pese a que esta especie es relativamente abundante en la zona pirenaica (Bueno Marí et al., 2009b), su prominente zoofilismo provoca que jamás haya sido relacionada con ningún episodio de transmisión de malaria en la literatura.

Examinando al principal vector detectado en la Comarca del Somontano de Barbastro, An. atroparvus, se ha demostrado que poblaciones europeas de esta especie son capaces de transmitir cepas asiáticas de $P$. vivax, pero son refractarias a cepas africanas de Plasmodium falciparum Welch,
1897 (Ramsdale \& Coluzzi 1975). Sin embargo, diversos estudios han identificado la capacidad de An. atroparvus de generar ooquistes de P. falciparum (Marchant et al. 1998), sin que haya podido ponerse en evidencia que es capaz de completar la esporogonia. Sin embargo, no debemos olvidar un hecho evidente, y es que el continuo contacto de $P$. falciparum, importado a nuestro país fundamentalmente por personas inmigrantes y turistas, con An. atroparvus, puede llevar a la selección y/o adaptación de distintas cepas de P. falciparum a desarrollarse en poblaciones locales de An. atroparvus. Cabe recordar que esta situación ya aconteció en el pasado, ya que durante el siglo XVIII, $A n$. atroparvus fue el principal responsable de continuas epidemias de fiebres tercianas malignas en diversos puntos de la Península Ibérica.

Pese a todas estas cuestiones que indican que la receptibilidad palúdica en la Comarca del Somontano de Barbastro es elevada debido a destacadas densidades de An. atroparvus en diversas zonas, cabe señalar que el potencial malariogénico global es relativamente bajo debido, fundamentalmente, a la baja vulnerabilidad palúdica de la comarca en particular y del país en general. La vulnerabilidad palúdica se refiere a la cantidad de plasmodios circulantes a la espera de proseguir su ciclo en el hospedador invertebrado, es decir, se basa en la población humana portadora. En este sentido, afortunadamente nuestro Sistema Nacional de Salud es muy férreo, ya que la malaria es una Enfermedad de Declaración Obligatoria (EDO). Ésta es una de las principales herramientas de las que disponemos para hacer frente a la enfermedad, ya que cualquier persona inmigrante o turista que llegue a España con paludismo debe ser sometida de manera obligatoria a los protocolos pertinentes de aislamiento y tratamiento de la enfermedad, puesto que en el ambiente circundante seguimos teniendo, como en el pasado, a excelentes vectores. A pesar de que todos los años se diagnostican alrededor de 400 casos de paludismo importado, los porcentajes son prácticamente nulos en la provincia de Huesca en los últimos 10 años. Sin embargo, también cabe señalar que se sabe que existe cierto porcentaje de infradiagnóstico en la enfermedad, fundamentalmente entre las personas inmigrantes. De hecho, pese a este bajo o prácticamente nulo porcentaje de paludismo importado en Huesca, en el año 2010 ocurrió en la Comarca de Monegros el primer caso de paludis- 
mo autóctono en España desde 1961. Sea como fuere, el hecho es que la vigilancia, tanto entomológica como epidemiológica, debe seguir fomentándose para minimizar la posible ocurrencia de casos como el acaecido en el año 2010.

\section{Agradecimientos}

Es voluntad del autor mostrar su más sincero agradecimiento al Centro de Estudios de Somontano de Barbastro (CESB) y a la Comisión de Cultura del Excelentísimo Ayuntamiento de Barbastro por la concesión XXII Concurso de Investigación López Novoa, que ha permitido la obtención de la financiación necesaria para el desarrollo del presente estudio. Asimismo, expresar también su gratitud al Instituto Aragonés de Gestión Ambiental (INAGA) por la expedición del permiso de captura de insectos en enclaves naturales de la comarca y agradecer la desinteresada colaboración mostrada por los Técnicos Medioambientales en su labor de asesoramiento acerca de potenciales puntos de muestreo a visitar. En este sentido, en especial destacar la ayuda prestada por D. Jesús Ruiz. Por último, también resaltar la ayuda recibida durante los muestreos por parte de diversos miembros y colaboradores del Laboratorio de Entomología y Control de Plagas de la Universitat de València, así como los interesantes y provechosos comentarios realizados por los revisores externos del presente trabajo.

\section{Referencias}

Alten B, Bellini R, Caglar SS, Simsek FM \& Kaynas S. 2000. Species composition and seasonal dynamic of mosquitoes in the Belek Region of Turkey. Journal of Vector Ecology 25: 146-154.

Aranda C, Panyella O, Erirtja R \& Castellà J. 1998. Canine filariasis Importance and transmission in the Baix Llobregat area, Barcelona (Spain). Veterinary Parasitology 77: 267-275.

Aranda C, Eritja R, Schaffner F \& Escosa R. 2000. Culex (Culex) torrentium Martini (Diptera: Culicidae) a new species from Spain. European Mosquito Bulletin 8: 7-9.

Bueno Marí R. 2010. Bioecología, diversidad e interés epidemiológico de los culícidos mediterráneos (Diptera, Culicidae). Valencia: Ed. Universitat de València.

Bueno Marí R, Moreno Marí J, Oltra Moscardí MT \& Jiménez Peydró R. 2009a. Artrópodos de interés vectorial en la Salud Pública española. Revista Española de Salud Pública 83: 199-212.

Bueno Marí R, Chordá Olmos FA, Bernués Bañeres A \& Jiménez Peydró R. 2009b. Aportaciones al conocimiento de los mosquitos (Diptera, Culicidae) de alta montaña presentes en la Península Ibérica. Pirineos 164: 49-68.

Bueno Marí R \& Jiménez Peydró R. 2008. Malaria en España: aspectos entomológicos y perspectivas de futuro. Revista Española de Salud Pública 82: 467489.

Bueno Marí R \& Jiménez Peydró R. 2010. New anopheline records from the Valencian Autonomous Region of Eastern Spain (Diptera: Culicidae: Anophelinae). European Mosquito Bulletin 28: 148-156.

Bueno Marí R, Bernués Bañeres A, Chordá Olmos FA \& Jiménez Peydró R. 2012a. Entomological surveillance in a recent autochthonous malaria area of Spain. Journal of Vector Borne Diseases 49: 45-46.

Bueno Marí R, Bernués Bañeres A \& Jiménez Peydró R. 2012b. Updated checklist and distribution maps of mosquitoes (Diptera: Culicidae) of Spain. European Mosquito Bulletin 30: 91-126.

Busquets N, Alba A, Allepuz A, Aranda C \& Nuñez JI. 2008. Usutu Virus sequences in Culex pipiens (Diptera: Culicidae), Spain. Emerging Infectious Diseases 14: 861-862.

Chippaux A. 1970. Hibernation de l'arbovirus Tahyna chez. Culex modestus Fic. en France. Les Comptes Rendus de l'Académie des Sciences 270: 16481650.

Darsie RF \& Saminadou Voyadjoglou A. 1997. Keys for the identification of the mosquitoes of Greece. Journal of American Mosquito Control Association 13: 247-254.

Edwards EW. A revision of the mosquitoes of the Paleartic Region. 1921. Bulletin of Entomological Research 12: 263-351.

Encinas Grandes A. 1982. Taxonomía y biología de los mosquitos del área salmantina (Diptera, Culicidae). Salamanca: Ed. Universidad de Salamanca.

Feng LC. 1938. A critical review of literature regarding the records of mosquitoes in China. Peking Natural History Bulletin 12: 139-181.

Gil Collado J. 1930. Datos actuales sobre la distribución geográfica de los Culícidos Españoles. Eos-Revista Española de Entomología 6: 329-347.

Gil Collado J. 1940. Sobre los biotipos (razas) españolas del Anopheles maculipenis. Revista de Sanidad e Higiene Pública 14: 26-32.

Guille G. 1976. Recherches éco-ethologiques sur Coquillettidiae (Coquillettidia) richardii (Ficalbi) 1889 (Diptera, Culicidae) du littoral méditerranéen francais. II. Millieu et comportament. Annales Des Sciences Naturelles Zoologie 18: 5-112.

Hackett LW \& Missiroli A. 1935. The varieties of Anopheles maculipennis and their relation to the distribution of malaria in Europe. Rivista di Malariologia 14: 45109.

Hannoun C. 1971. Progrès recénts dans l'étudde des arbovirus. Bulletin de l'Institute Pasteur 69: 241-278.

Horsfall WR. 1972. Mosquitoes: their bionomics and relation to disease. New York: Ed. Hafner Publications.

Jordá Llona JR. 1993. Culícidos (Diptera, Culicidae) del Parque Nacional de Doñana (SW de España). Aspectos faunísticos y ecológicos. Anales de Biología 19: 93-104. 
Lachmajer J. 1971. Biology of Anopheles claviger (Meigen, 1804) populations (Diptera, Culicinae) in the Gdansk environment. Acta Parasitologica Polonica 14: 163-184.

Llave Correas C \& González Mora D. 1996. Los mosquitos (Diptera, Culicidae) en las viviendas de Madrid. Boletín de la Real Sociedad Española de Historia Natural (Sección Biología) 92: 215-220.

López Sánchez S. 1989. Control integral de mosquitos en Huelva. Sevilla: Ed. Junta de Andalucía. Conserjería de Salud y Servicios Sociales.

Lozano A. 1953. El estadio invernante del Anopheles maculipennis atroparvus y su relación con la pausa estacional del paludismo en España. Revista de Sanidad e Higiene Pública 27: 303-325.

Marchant P, Rling W, Van Gemert GJ, Leake CJ \& Curtis CF. 1998. Could british mosquitoes transmit falciparum malaria? Parassitology Today 14: 344-345.

Minar J. 1969. A contribution on the bionomy of Culex modestus Fic. (Diptera, Culicidae) in Southern Moravia. Folia Parasitologica 16: 93-96.

Mouchet J, Rageau J, Laumond C, Hannoun C, Beytout D, Ondar J., Corniou B \& Chippaux A. 1970. Epidémiologie du virus West Nile: etude d'un foyer en Camargue. V. Le vecteur: Culex modestus Ficalbi (Diptera, Culicidae.). Annales I'Institute Pasteur 118: 839-855.

Moussiegt O. 1990. Culex (Barradius) modestus Ficalbi, 1889. Bibliographie. Entente Interdepartementale de Demoustication du Littoral Méditerranéenne 59: 135.

Nieto Callén JJ \& Bosch Ferrer JR. 1991. La epidemia de tercianas de 1783-85 en Barbastro y su corregimiento: miseria, mortandad y asistencia a finales del antiguo régimen. Somontano 2: 63-83.

Ramsdale CD \& Coluzzi M. 1975. Studies on the infectivity of tropical African strains of Plasmodium falciparum to some southern European vectors of malaria. Parassitologia 17: 39-48.

Rioux JA. 1958. Les Culicidés du Midi méditerranéen. Encyclopédie Entomologique XXXV. Paris: Ed. Paul Lechevalier.

Romi R, Pontuale G \& Sabatinelli G. 1997. Le zanzareitaliane: generalità e identificazione degli stadi preimaginali (Diptera: culicidae). Fragmenta entomologica 29: 1-141.

Santa-Olalla Peralta $P$, Vázquez-Torres MC, Latorre-Fandós E, Mairal-Claver, $P$, Cortina-Solano P., Puy-Azón A, Adiego-Sancho B, Leitmeyer K, Lucientes- Curdi, J \& Sierra-Morós MJ. 2010. First autochthonous malaria case due to Plasmodium vivax since eradication, Spain, October 2010. Euro Surveillance 15(41):pii=19684.Disponible en: http:// www.eurosurveillance.org/ViewArticle.aspx?Articleld $=19684$

Schäefer M. 2004. Mosquitoes as a Part of Wetland Biodivesity. Uppsala: Ed. Acta Universitatis Upsaliensis.

Schaffner F, Angel G, Geoffroy B, Hervy JO \& Rhaeim A. 2001. The mosquitoes of Europe / Les moustiques d' Europe. Montpellier, France: IRD Éditions and EID Méditerranée.

Sicart M. 1951. Note sur la présence de Culex mimeticus Noè 1899, en Tunisie. Bulletin Society of Sciences Naturals Tunisie 4: 60-61. 


\section{Apéndice}

Listado de las especies halladas con indicación de las coordenadas geográficas, altitud y población de sus biotopos larvarios detectados

\begin{tabular}{|c|c|c|c|c|c|}
\hline Especie & Latitud & Longitud & Altitud & Población & $\mathrm{N}^{\circ}$ ejemplares \\
\hline An. atroparvus & $42^{\circ} 08^{\prime} 59.1^{\prime \prime}$ & $0^{\circ} 01^{\prime} 40.7$ 'E & 463 & Alquézar & 14 \\
\hline An. atroparvus & $41^{\circ} 56^{\prime} 49.8^{\prime \prime}$ & $0^{\circ} 02$ '05.2”O & 409 & Berbegal & 5 \\
\hline An. atroparvus & $42^{\circ} 15^{\prime} 01.2 "$ & $0^{\circ} 05^{\prime} 13.2 " 0$ & 637 & Pedruel (Bierge) & 8 \\
\hline An. atroparvus & $42^{\circ} 08^{\prime} 23.3^{\prime \prime}$ & 001'31.3"'E & 447 & Buera (Santa María de Dulcis) & 5 \\
\hline An. atroparvus & $41^{\circ} 58^{\prime} 12.6 ”$ & 010'15.3"'E & 273 & Castejón del Puente & 6 \\
\hline An. atroparvus & 41ํ5'11.1" & 010'12.4'"E & 266 & Castejón del Puente & 3 \\
\hline An. atroparvus & $42^{\circ} 10^{\prime} 26.2^{\prime \prime}$ & 004'26.5'” & 628 & Colungo & 2 \\
\hline An. atroparvus & $42^{\circ} 07^{\prime} 09.5^{\prime \prime}$ & 0०13'47.1'” & 355 & El Grado & 9 \\
\hline An. atroparvus & $42^{\circ} 07^{\prime} 10.8^{\prime \prime}$ & 0013'49.5'E & 372 & El Grado & 7 \\
\hline An. atroparvus & 4203'54.3"' & 012'58.5”'E & 323 & Estada & 13 \\
\hline An. atroparvus & 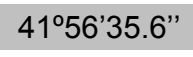 & 003'36.6"'E & 301 & Monesma (Ilche) & 45 \\
\hline An. atroparvus & 415'ㄷ‥' & $0^{\circ} 03^{\prime} 42.8^{\prime \prime} \mathrm{E}$ & 306 & Monesma (llche) & 12 \\
\hline An. atroparvus & 415'42.2"' & 004'03.6"'E & 315 & Permisán (llche) & 7 \\
\hline An. atroparvus & 42011'28.9"' & 009'14.6”'E & 568 & Naval & 10 \\
\hline An. atroparvus & 42011'11.4"' & 009'15.1'E & 557 & Naval & 11 \\
\hline An. atroparvus & 415'22.7'" & 005'37.8”O & 348 & Peralta de Alcofea & 9 \\
\hline An. maculipennis & $42^{\circ} 08^{\prime} 47.9^{\prime \prime}$ & 003'34.2"O & 565 & Abiego & 6 \\
\hline An. maculipennis & $42^{\circ} 08^{\prime} 59.1^{\prime \prime}$ & 001'40.7'” & 463 & Alquézar & 5 \\
\hline An. maculipennis & $42^{\circ} 09^{\prime} 54.4^{\prime \prime}$ & 001'36.3"'E & 479 & Alquézar & 7 \\
\hline An. maculipennis & 42¹5’01.2”' & 005'13.2"O & 637 & Pedruel (Bierge) & 3 \\
\hline An. maculipennis & 4208'23.3"' & 001'31.3”'E & 447 & Buera & 6 \\
\hline An. maculipennis & 4207'10.8' & 0०13'49.5'” & 372 & El Grado & 2 \\
\hline An. maculipennis & $42^{\circ} 03^{\prime} 54.3^{\prime \prime}$ & 012'58.5”E & 323 & Estada & 4 \\
\hline An. maculipennis & $42^{\circ} 04^{\prime} 39.1^{\prime \prime}$ & 002'14.6”'E & 375 & Pozán de Vero & 9 \\
\hline An. petragnani & $42^{\circ} 09^{\prime} 54.0^{\prime \prime}$ & 001'59.1"O & 372 & Alberuela de la Liena (Abiego) & 6 \\
\hline An. petragnani & $42^{\circ} 14^{\prime} 09.9^{\prime \prime}$ & $0^{\circ} 02{ }^{\prime} 37.5^{\prime \prime} \mathrm{O}$ & 672 & Adahuesca & 9 \\
\hline An. petragnani & $42^{\circ} 10^{\prime} 28.1^{\prime \prime}$ & $0^{\circ} 03^{\prime 2} 25.1$ '” & 574 & Asque (Colungo) & 12 \\
\hline Cs. annulata & $42^{\circ} 10^{\prime} 36.2^{\prime \prime}$ & 000’51.3"O & 620 & Radiquero (Alquézar) & 4 \\
\hline Cs. longiareolata & $42^{\circ} 10^{\prime} 03.5^{\prime \prime}$ & 002'15.1"O & 540 & Alberuela de la Liena (Abiego) & 12 \\
\hline Cs. longiareolata & $42^{\circ} 10^{\prime} 34.6^{\prime \prime}$ & 001'04.8'E & 777 & Alquézar & 9 \\
\hline Cs. longiareolata & $42^{\circ} 10^{\prime} 36.2^{\prime \prime}$ & 000'51.3"O & 620 & Radiquero (Alquézar) & 19 \\
\hline Cs. longiareolata & 4211'26.2”' & 000'29.9'” & 875 & San Pelegrín (Alquézar) & 13 \\
\hline Cs. longiareolata & $42^{\circ} 02^{\prime} 07.1^{\prime \prime}$ & 007'38.2'"E & 518 & Barbastro & 15 \\
\hline Cs. longiareolata & $42^{\circ} 08^{\prime} 49.8^{\prime \prime}$ & 004'28.7'O & 593 & Bierge & 14 \\
\hline Cs. longiareolata & $42^{\circ} 10^{\prime} 30.3^{\prime \prime}$ & 003'22.2'"E & 591 & Asque (Colungo) & 1 \\
\hline Cs. longiareolata & $42^{\circ} 10^{\prime} 47.3^{\prime \prime}$ & 004'01.5”'E & 647 & Colungo & 6 \\
\hline Cs. longiareolata & $42^{\circ} 10^{\prime} 46.8^{\prime \prime}$ & 004'01.1'"E & 645 & Colungo & 21 \\
\hline Cs. longiareolata & $42^{\circ} 10^{\prime} 03.8^{\prime \prime}$ & 07'17.9'” & 743 & Salinas de Hoz (Hoz y Costeán) & 11 \\
\hline Cs. longiareolata & $42^{\circ} 01^{\prime} 06.9^{\prime \prime}$ & 002'18.2”O & 455 & Laluenga & 10 \\
\hline Cx. hortensis & $42^{\circ} 08^{\prime} 39.8^{\prime \prime}$ & 004'19.1"O & 581 & Abiego & 7 \\
\hline Cx. hortensis & $42^{\circ} 14^{\prime} 09.9^{\prime \prime}$ & $0^{\circ} 02 ' 37.5 ” O$ & 672 & Adahuesca & 9 \\
\hline Cx. hortensis & $42^{\circ} 10^{\prime} 46.8^{\prime \prime}$ & 004'01.1'” & 645 & Colungo & 7 \\
\hline Cx. impudicus & $42^{\circ} 09^{\prime} 53.1^{\prime \prime}$ & 002'20.1'” & 553 & Alberuela de la Liena (Abiego) & 9 \\
\hline Cx. impudicus & $42^{\circ} 10^{\prime} 31.6^{\prime \prime}$ & $0^{\circ} 00^{\prime} 47.3 " \mathrm{O}$ & 608 & Radiquero (Alquézar) & 6 \\
\hline Cx. impudicus & $42^{\circ} 10^{\prime} 36.2^{\prime \prime}$ & 000'51.3"O & 620 & Radiquero (Alquézar) & 3 \\
\hline
\end{tabular}




\begin{tabular}{|c|c|c|c|c|c|}
\hline Especie & Latitud & Longitud & Altitud & Población & $\mathrm{N}^{\circ}$ ejemplares \\
\hline Cx. impudicus & $42^{\circ} 04^{\prime} 05.2^{\prime \prime}$ & $0^{\circ} 05^{\prime} 14.7{ }^{\prime \prime} \mathrm{E}$ & $360 \mathrm{E}$ & Barranco del Ariño (Barbastro) & 2 \\
\hline Cx. impudicus & 4158'12.6”" & 010'15.3"'E & 273 & Castejón del Puente & 3 \\
\hline Cx. impudicus & $42^{\circ} 12^{\prime} 47.9^{\prime \prime}$ & 012'37.7'” & $487 \quad$ & Mipanas (Naval) & 14 \\
\hline Cx. mimeticus & $42^{\circ} 10^{\prime} 02.5^{\prime \prime}$ & 002'19.3"O & 561 & Alberuela de la Liena (Abiego) & 8 \\
\hline Cx. mimeticus & $42^{\circ} 09^{\prime} 52.6 "$ & $0^{\circ} 02 ' 13.2 " \mathrm{O}$ & 518 & Alberuela de la Liena (Abiego) & 6 \\
\hline Cx. mimeticus & $42^{\circ} 10^{\prime} 31.6^{\prime \prime}$ & $0^{\circ} 00^{\prime} 47.3 " \mathrm{O}$ & $608 \mathrm{~F}$ & Radiquero (Alquézar) & 11 \\
\hline Cx. mimeticus & $42^{\circ} 11^{\prime} 26.9^{\prime \prime}$ & 000'29.3”'E & $872 \leqslant$ & San Pelegrín (Alquézar) & 15 \\
\hline Cx. mimeticus & $42^{\circ} 02^{\prime} 07.9^{\prime \prime}$ & $0^{\circ} 07^{\prime} 41.1$ 'E & 509 & Barbastro & 2 \\
\hline Cx. mimeticus & $42^{\circ} 10^{\prime} 28.1^{\prime \prime}$ & 003'25.1'” & 574 & Asque (Colungo) & 14 \\
\hline Cx. mimeticus & $42^{\circ} 04^{\prime} 39.1^{\prime \prime}$ & 002'14.6”'E & $375 \mathrm{~F}$ & Pozán de Vero & 11 \\
\hline Cx. modestus & $41^{\circ} 56^{\prime} 56.9^{\prime \prime}$ & 003'42.8'"E & 3061 & Monesma (Ilche) & 9 \\
\hline Cx. pipiens & $42^{\circ} 09^{\prime} 53.8^{\prime \prime}$ & $0^{\circ} 02 ' 13.7 " \mathrm{O}$ & 515 & Alberuela de la Liena (Abiego) & 5 \\
\hline Cx. pipiens & $42^{\circ} 10^{\prime} 03.5^{\prime \prime}$ & $0^{\circ} 02 ' 15.1 " \mathrm{O}$ & 540 & Alberuela de la Liena (Abiego) & 139 \\
\hline Cx. pipiens & $42^{\circ} 09^{\prime} 53.1^{\prime \prime}$ & $0^{\circ} 02 ' 20.1 " \mathrm{O}$ & $553 r$ & Alberuela de la Liena (Abiego) & 2 \\
\hline Cx. pipiens & $42^{\circ} 08^{\prime} 59.1^{\prime \prime}$ & 001'40.7'” & 463 & Alquézar & 29 \\
\hline Cx. pipiens & $42^{\circ} 10^{\prime} 03.9^{\prime \prime}$ & 001'37.9'"E & 543 & Alquézar & 21 \\
\hline Cx. pipiens & $42^{\circ} 10^{\prime} 34.6^{\prime \prime}$ & 001'04.8'E & $777 f$ & Alquézar & 39 \\
\hline Cx. pipiens & $42^{\circ} 10^{\prime} 31.6^{\prime \prime}$ & $0^{\circ} 00^{\prime} 47.3 " \mathrm{O}$ & $608 \mathrm{~F}$ & Radiquero (Alquézar) & 1 \\
\hline Cx. pipiens & $42^{\circ} 10^{\prime} 36.2^{\prime \prime}$ & $0^{\circ} 00^{\prime} 51.3 " 0$ & $620 \mathrm{~F}$ & Radiquero (Alquézar) & 38 \\
\hline Cx. pipiens & $42^{\circ} 11^{\prime} 26.2^{\prime \prime}$ & 000'29.9'” & $875 \leqq$ & San Pelegrín (Alquézar) & 28 \\
\hline Cx. pipiens & $42^{\circ} 02^{\prime} 07.9^{\prime \prime}$ & $0^{\circ} 07$ '41.1'” & 509 & Barbastro & 2 \\
\hline Cx. pipiens & $42^{\circ} 02^{\prime} 07.1^{\prime \prime}$ & 007'38.2'” & 518 & Barbastro & 8 \\
\hline Cx. pipiens & $42^{\circ} 02^{\prime} 11.1^{\prime \prime}$ & $0^{\circ} 05^{\prime} 12.8 \prime \mathrm{O}$ & 489 & Barbuñales & 8 \\
\hline Cx. pipiens & $42^{\circ} 08^{\prime} 49.8^{\prime \prime}$ & 004'28.7'O & 593 & Bierge & 2 \\
\hline Cx. pipiens & 4158'12.6”' & 0०10'15.3”'E & 273 & Castejón del Puente & 2 \\
\hline Cx. pipiens & $42^{\circ} 10^{\prime} 30.3^{\prime \prime}$ & 003'22.2'” & 591 & Asque (Colungo) & 24 \\
\hline Cx. pipiens & $42^{\circ} 10^{\prime} 26.2^{\prime \prime}$ & $0^{\circ} 04^{\prime} 26.5^{\prime \prime} \mathrm{E}$ & 628 & Colungo & 2 \\
\hline Cx. pipiens & $42^{\circ} 10^{\prime} 03.8^{\prime \prime}$ & 07'17.9'” & $743 \leqq$ & Salinas de Hoz (Hoz y Costeán) & 33 \\
\hline Cx. pipiens & $41^{\circ} 58^{\prime} 35.6 "$ & $0^{\circ} 03$ '33.7'” & $314 F$ & Fornillos (Ilche) & 3 \\
\hline Cx. pipiens & $41^{\circ} 56^{\prime} 35.6^{\prime \prime}$ & 003'36.6”'E & 3011 & Monesma (Ilche) & 8 \\
\hline Cx. pipiens & $41^{\circ} 56^{\prime} 56.9^{\prime \prime}$ & 003'42.8'” & 3061 & Monesma (Ilche) & 3 \\
\hline Cx. pipiens & $42^{\circ} 01^{\prime} 06.9^{\prime \prime}$ & 002'18.2"O & $455 \mathrm{~L}$ & Laluenga & 7 \\
\hline Cx. pipiens & $42^{\circ} 12^{\prime} 47.9^{\prime \prime}$ & 012'37.7'” & 4871 & Mipanas (Naval) & 13 \\
\hline Cx. pipiens & $41^{\circ} 55^{\prime} 22.7^{\prime \prime}$ & $0^{\circ} 05^{\prime} 37.8^{\prime \prime} \mathrm{O}$ & $348 F$ & Peralta de Alcofea & 16 \\
\hline Cx. pipiens & $42^{\circ} 04^{\prime} 39.1^{\prime \prime}$ & 002'14.6”'E & $375 \mathrm{~F}$ & Pozán de Vero & 1 \\
\hline Cx. pipiens & $42^{\circ} 14^{\prime} 19.4^{\prime \prime}$ & 011'27.2'"E & $686 r$ & Abizanda (Comarca Sobrarbe) & 43 \\
\hline Cx. territans & $42^{\circ} 09^{\prime} 53.1^{\prime \prime}$ & 002'20.1'” & 553 & Alberuela de la Liena (Abiego) & 16 \\
\hline Cx. territans & $42^{\circ} 09^{\prime} 15.2^{\prime \prime}$ & 001'26.3”'E & 510 & Alquézar & 4 \\
\hline Cx. territans & $42^{\circ} 10^{\prime} 31.6 "$ & $0^{\circ} 00^{\prime} 47.3 " 0$ & $608 \mathrm{~F}$ & Radiquero (Alquézar) & 8 \\
\hline Cx. territans & $42^{\circ} 10^{\prime} 36.2^{\prime \prime}$ & 000'51.3"O & $620 \mathrm{~F}$ & Radiquero (Alquézar) & 9 \\
\hline Cx. territans & $42^{\circ} 04^{\prime} 05.2^{\prime \prime}$ & 005'14.7'” & $360 \mathrm{E}$ & Barranco del Ariño (Barbastro) & 5 \\
\hline Cx. territans & $42^{\circ} 08^{\prime} 23.3^{\prime \prime}$ & 001'31.3'” & $447 \quad E$ & Buera (Santa María de Dulcis) & 3 \\
\hline Cx. territans & 415'12.6"' & 010'15.3”'E & 273 & Castejón del Puente & 7 \\
\hline Cx. territans & $42^{\circ} 10^{\prime} 26.2^{\prime \prime}$ & $0^{\circ} 04^{\prime} 26.5$ "O & 628 & Colungo & 2 \\
\hline Cx. territans & $42^{\circ} 12^{\prime} 47.9^{\prime \prime}$ & 012'37.7'” & 4871 & Mipanas (Naval) & 12 \\
\hline Cx. territans & $42^{\circ} 14^{\prime} 19.4^{\prime \prime}$ & 011'27.2'"E & $686 r$ & Abizanda (Comarca Sobrarbe) & 8 \\
\hline Cx. terrritans & $42^{\circ} 10^{\prime} 30.3^{\prime \prime}$ & 003'22.2'” & 591 & Asque (Colungo) & 11 \\
\hline Oc. caspius & $41^{\circ} 57^{\prime} 42.2^{\prime \prime}$ & 004'03.6”'E & $315 \mathrm{~F}$ & Permisán (Ilche) & 48 \\
\hline
\end{tabular}

